MATHEMATICS OF COMPUTATION

Volume 71, Number 237, Pages 415-427

S 0025-5718(00)01292-8

Article electronically published on October 18, 2000

\title{
UPPER BOUNDS FOR THE PRIME DIVISORS OF WENDT'S DETERMINANT
}

\author{
ANASTASIOS SIMALARIDES
}

\begin{abstract}
Let $c \geq 2$ be an even integer, $(3, c)=1$. The resultant $W_{c}$ of the polynomials $t^{c}-1$ and $(1+t)^{c}-1$ is known as Wendt's determinant of order $c$. We prove that among the prime divisors $q$ of $W_{c}$ only those which divide $2^{c}-1$ or $L_{c / 2}$ can be larger than $\theta^{c / 4}$, where $\theta=2.2487338$ and $L_{n}$ is the $n$th Lucas number, except when $c=20$ and $q=61$. Using this estimate we derive criteria for the nonsolvability of Fermat's congruence.
\end{abstract}

\section{INTRODUCTION}

Let $c \geq 2$ be an even integer. Given two polynomials $f(t)$ and $g(t)$ denote by $R(f(t), g(t))$ their resultant. The integer

$$
W_{c}=R\left(t^{c}-1,(1+t)^{c}-1\right)
$$

is known as Wendt's determinant. The prime divisors of $W_{c}$ are of importance because of the following result of Wendt [16].

Theorem 1. Let $p, q$ be odd primes such that $q=1+c p,(3, c)=1$. Then, Fermat's congruence

$$
x^{p}+y^{p}+z^{p} \equiv 0 \quad(\bmod q)
$$

has a nontrivial solution (that is, a solution $(x, y, z)$ such that $x y z \not \equiv 0(\bmod q))$ if and only if $q$ divides $W_{c}$.

Although Fermat's Problem has been solved completely, some questions concerning congruence (1I) (or, equivalently, the number $W_{c}$ ) remain still unanswered (cf. Section 5).

Since $W_{c}=0$ if and only if $(3, c)>1$, we shall assume through the paper that $(3, c)=1$. The quantity $\left|W_{c}\right|$ grows rapidly with $c$; Boyd [1] proved that

$$
10^{-1 / 3} \lambda^{c^{2}}<\left|W_{c}\right|<10^{1 / 3} \lambda^{c^{2}},
$$

where $\log \lambda=\frac{2}{\pi} \int_{0}^{\pi / 3} \log (2 \cos \theta) d \theta=0.323 \ldots$ In the Table 1 below we list the first few values of $\left|W_{c}\right|$. Several authors carried out the complete factorization of $W_{c}$ for $c \leq c_{0}$ : Frame [8] for $c_{0}=50$; Fee and Granville [6] for $c_{0}=200$; Ford and Jha [7] for $c_{0}=500$.

Received by the editor April 13, 1999 and, in revised form, February 24, 2000.

2000 Mathematics Subject Classification. Primary 11C20; Secondary 11Y40, 11D79.

Key words and phrases. Wendt's determinant, Fermat's congruence. 
TABLE 1. The values of $\left|W_{c}\right|$ for $c \leq 20$

\begin{tabular}{|c|l|c|l|}
\hline$c$ & $\left|W_{c}\right|$ & $c$ & $\left|W_{c}\right|$ \\
\hline 2 & 3 & 14 & $2^{24} \cdot 3 \cdot 29^{6} \cdot 43^{3} \cdot 127^{3}$ \\
\hline 4 & $3 \cdot 5^{3}$ & 16 & $3^{7} \cdot 5^{3} \cdot 7^{6} \cdot 17^{15} \cdot 257^{3}$ \\
\hline 8 & $3^{7} \cdot 5^{3} \cdot 17^{3}$ & 20 & $3 \cdot 5^{24} \cdot 11^{9} \cdot 31^{3} \cdot 41^{9} \cdot 61^{6}$ \\
\hline 10 & $3 \cdot 11^{9} \cdot 31^{3}$ & & \\
\hline
\end{tabular}

By the well-known factorizations (cf. [8])

$$
\begin{aligned}
W_{c} & =\prod_{a=1}^{c} \prod_{b=1}^{c}\left(1+\zeta^{a}+\zeta^{b}\right) \\
& =\prod_{a=1}^{c} \prod_{b=1}^{c}\left(1-\zeta^{a}-\zeta^{b}\right), \quad \zeta=e^{2 \pi i / c},
\end{aligned}
$$

of $W_{c}$, it follows immediately that the integer $2^{c}-1$ divides $W_{c}$. It follows also in an analogous way (cf. Section 2) that $L_{c / 2}$ divides $W_{c}$ ( $L_{n}$ is the $n$th Lucas number), in case $c \equiv 2(\bmod 4)$.

Such nice factors of $W_{c}$ are called principal factors. Further information on the principal factors of $W_{c}$ can be found in E. Lehmer [1], Frame [8] and Ribenboim [12]; for a recent result see Helou [9]. The factorization of the principal factors

$$
2^{c}-1, \quad L_{c / 2},
$$

is of special importance, because the greatest prime divisor of $W_{c}$ divides often one of the numbers (31). The extensive tables by Brillhart et al. [2], contain all the known factorizations of the numbers $2^{c}-1$ for $c \leq 2400$; other tables by Brillhart et al. 3] contain all the known factorizations of the Lucas numbers $L_{n}$ for $n \leq 500$. Unfortunately, no complete factorization of $W_{c}$ is known that involves only simple principal factors.

Upper bounds for the prime divisors of $W_{c}$ are obtained in the following way. Let $q$ be a prime divisor of $W_{c}$, which does not divide $c$. It follows by (2) that a prime ideal divisor of $q$ in $\mathcal{Q}(\zeta)$ divides a trinomial cyclotomic integer $1+\zeta^{a}+\zeta^{b}$. In consequence, $q$ divides both the norm

$$
N=N(a, b)=N_{\mathcal{Q}(\zeta) / \mathcal{Q}}\left(1+\zeta^{a}+\zeta^{b}\right)
$$

of $1+\zeta^{a}+\zeta^{b}$ and the resultant

$$
R=R(a, b)=R\left(1+t^{a}+t^{b}, t^{c / 2}+1\right)
$$

of the polynomials $1+t^{a}+t^{b}$ and $t^{c / 2}+1$; in consequence, it suffices to estimate one of the numbers $|N|$ and $|R|$. Bounds which arise from the estimation of $|N|$ have their origin in Vandiver [15, who first noticed and used the simplest possible estimate $|N| \leq 3^{\phi(c)}$ of this type ( $\phi$ is Euler's function). Improved bounds of this type were proved and used by Denes [5], Simalarides [13], and, Fee and Granville [6]. Bounds that arise from the estimation of $|R|$ have their origin in Krasner [10], who proved that $q \leq 3^{c / 4}$ for every prime divisor $q$ of $W_{c}$ such that $2^{c} \not \equiv 1(\bmod q)$ and $q=1+c p$, where $p$ is a prime. The author [14 improved upon Krasner's result by proving that $q \leq 3+(2.618 \ldots)^{c / 4}$, under the same conditions. In the same paper, it was also proved that $q \leq 2.459^{c / 4}$ under the additional condition 
that $q$ does not divide the numbers $1+(-1)^{c / 2} \pm L_{c / 2}$. The results in [10] and [14] were not formulated explicitly as results concerning the resultant $W_{c}$, but rather, as results concerning the first case of Fermat's Last Theorem.

We generalize and improve all these previous results as follows.

Theorem 2. Let $c \geq 2$ be an even integer such that $(3, c)=1$. If a prime divisor $q$ of $W_{c}$ satisfies the inequality

$$
q>\theta^{c / 4}, \text { where } \theta=2.2487338,
$$

then at least one of the following is true: (i) $c=20$ and $q=61$; (ii) $q$ is a divisor of $2^{c}-1$; (iii) $c \equiv 2(\bmod 4)$ and $q$ is a divisor of $L_{c / 2}$.

The proof of Theorem 2 will be given in Section 3 .

In case $c \equiv 0(\bmod 4)$ the number $2^{c}-1$ admits the obvious factorization

$$
2^{c}-1=\left(2^{c / 4}-1\right)\left(2^{c / 4}+1\right)\left(2^{c / 2}+1\right),
$$

while in case $c \not \equiv 0(\bmod 8)$, it can be factored further (Aurifeuillian factorization) as follows:

$$
2^{c}-1=\left(2^{c / 4}-1\right)\left(2^{c / 4}+1\right)\left(2^{c / 4}-2^{(c+4) / 8}+1\right)\left(2^{c / 4}+2^{(c+4) / 8}+1\right) .
$$

In view of these factorizations, Theorem 2 can be written in the following sharper form.

Theorem 3. Let $c \geq 2$ be an even integer such that $(3, c)=1$. Then, among the prime divisors $q$ of $W_{c}$, only those which divide either

$$
2^{c}-1 \text { or } L_{c / 2}, \quad \text { in case } c \equiv 2 \quad(\bmod 4),
$$

or

$$
2^{c / 2}+1, \quad \text { in case } c \equiv 0 \quad(\bmod 8),
$$

can be larger than $\theta^{c / 4}$, where $\theta=2.2487338$, except when

$$
(c, q) \in\{(4,3),(4,5),(20,61)\} .
$$

\section{Preliminaries concerning Fibonacci and Lucas numbers}

The formulae

$$
L_{2 n}=L_{n}^{2}-2(-1)^{n}, \quad 4+L_{2 n-1}^{2}=5 F_{2 n-1}^{2}, \quad n \geq 1,
$$

are immediate consequences of the standard expresssions

$$
L_{n}=\omega_{1}^{n}+\omega_{2}^{n}, \quad F_{n}=\frac{\omega_{2}^{n}-\omega_{1}^{n}}{\omega_{2}-\omega_{1}}, \quad n \geq 1
$$

for the $n$th Lucas and Fibonacci numbers, respectively, where $\omega_{1}=(1-\sqrt{5}) / 2$, $\omega_{2}=(1+\sqrt{5}) / 2$, are the roots of the polynomial $t^{2}-t-1$. Define

$$
u_{c}=R\left(t^{2}+t-1, t^{c}-1\right) .
$$

The following lemma shows that $u_{c}$ is a principal factor of $W_{c}$. 
Lemma 1. Let $c \geq 2$ be an even integer such that $(3, c)=1$. Then the following hold true:

(i) The integer $u_{c}$ is a divisor of $W_{c}$.

(ii) We have

$$
\begin{aligned}
u_{c}=2-L_{c}=2+2(-1)^{c / 2}-L_{c / 2}^{2} \\
= \begin{cases}\left(2-L_{\frac{c}{4}}\right)\left(2+L_{\frac{c}{4}}\right) L_{\frac{c}{4}}^{2} & \text { if } c \equiv 0 \quad(\bmod 8), \\
-5 F_{\frac{c}{4}}^{2} L_{\frac{c}{4}}^{2} & \text { if } c \equiv 4 \quad(\bmod 8), \\
-L_{\frac{c}{2}}^{2} & \text { if } c \equiv \pm 2 \quad(\bmod 8) .\end{cases}
\end{aligned}
$$

(iii) If a prime divisor $q \neq 5$ of $u_{c}$ is larger than $\theta^{c / 4}$, then $c \equiv 2(\bmod 4)$ and $q$ is a divisor of $L_{c / 2}$.

Proof. (i) Immediate in view of (2) and the fact that

$$
u_{c}=\prod_{a=1}^{c}\left(\zeta^{2 a}+\zeta^{a}-1\right) .
$$

(ii) We have

$$
\begin{aligned}
u_{c}=\left(\omega_{1}^{c}-1\right)\left(\omega_{2}^{c}-1\right) & =\left(\omega_{1} \omega_{2}\right)^{c}-\left(\omega_{1}^{c}+\omega_{2}^{c}\right)+1 \\
& =2-L_{c} .
\end{aligned}
$$

Applying formulae (5) we obtain the rest of the result sought.

(iii) Immediate in view of (ii) and of the obvious bounds

$$
L_{n} \leq 1+\omega_{2}^{n}=1+(1.618 \ldots)^{n}, \quad F_{n} \leq \frac{\omega_{2}^{n}+1}{\sqrt{5}}=\frac{(1.618 \ldots)^{n}+1}{\sqrt{5}},
$$

where $n \geq 1$.

\section{Proof of Theorem 2}

First of all, Theorem 2 is true for $c \leq 20$, so we can assume that $c \geq 22$. Assume that there is a prime divisor $q$ of $W_{c}$ which satisfies the inequality (4). Assume also that $q$ is neither a divisor of $2^{c}-1$, nor a divisor of $L_{c / 2}$ in case $c \equiv 2(\bmod 4)$. We shall prove that this assumption leads to a contradiction. Hypothesis (4) implies that $q>c$, so $q$ does not divide $c$; it follows that

$$
1+\zeta^{a}+\zeta^{b} \equiv 0 \quad(\bmod \mathbf{q})
$$

where $\mathbf{q}$ is a prime ideal divisor of $q$ in $\mathcal{Q}(\zeta)$, and $a, b$ are two integers such that

$$
a \not \equiv 0, \quad b \not \equiv 0, \quad a \not \equiv b \quad(\bmod c)
$$

(the last three relations are immediate consequences of the hypothesis $2^{c} \not \equiv 1$ $(\bmod q))$.

Since $\zeta^{c / 2}+1=0$, the resultant $R(a, b)$ of the polynomials $1+t^{a}+t^{b}, t^{c / 2}+1$ satisfies the congruence

$$
R(a, b) \equiv 0 \quad(\bmod q) .
$$

We can assume that $q \equiv 1(\bmod c)$; otherwise would have $R(a, b) \equiv 0\left(\bmod q^{2}\right)$, and in consequence $q<3^{c / 8}$, which would contradict hypothesis (4). 
The integer $R(a, b)$ admits the following representation:

$$
\begin{aligned}
R(a, b)= & \prod_{i=1}^{c / 2}\left[1+\zeta^{(2 i-1) a}+\zeta^{(2 i-1) b}\right] \\
= & \prod_{i=1}^{c_{1}}\left[3+2 \cos \frac{2 \pi a}{c}(2 i-1)\right. \\
& \left.\quad+2 \cos \frac{2 \pi b}{c}(2 i-1)+2 \cos \frac{2 \pi(a-b)}{c}(2 i-1)\right] d
\end{aligned}
$$

where

$$
c_{1}=\left\{\begin{array}{cl}
\frac{c}{4} & \text { if } c \equiv 0 \quad(\bmod 4) \\
\frac{c}{4}-\frac{1}{2} & \text { if } c \neq \equiv 0 \quad(\bmod 4)
\end{array}\right.
$$

and

$$
d=\left\{\begin{array}{ccc}
1 & \text { if } c \equiv 0 \quad(\bmod 4) \\
1+(-1)^{a}+(-1)^{b} & \text { if } c \neq \equiv 0 & (\bmod 4)
\end{array}\right.
$$

We have $R(a, b) \neq 0$ because of the relation $(3, c)=1$. Introducing the abbreviation

$$
A_{i}=\cos \frac{2 \pi a}{c}(2 i-1)+\cos \frac{2 \pi b}{c}(2 i-1)+\cos \frac{2 \pi(a-b)}{c}(2 i-1),
$$

we obtain

$$
\log |R(a, b)|=\sum_{i=1}^{c_{1}} \log \left(3+2 A_{i}\right)+\log |d|,
$$

where evidently $-1.5<A_{i} \leq 3$. We have

$$
\log (3+2 z)<\sum_{j=0}^{4} \alpha_{j} z^{j}, \text { for }-1.5<z \leq 3,
$$

where $\alpha_{0}=1.166985006, \alpha_{1}=0.76146, \alpha_{2}=-0.295509605, \alpha_{3}=0.0523446$, $\alpha_{4}=0.0014453$. This implies that

$$
\log |R(a, b)|<\sum_{i=1}^{c_{1}} \sum_{j=0}^{4} \alpha_{j} A_{i}^{j}+\log |d|=\sum_{j=0}^{4} \alpha_{j} \sum_{i=1}^{c_{1}} A_{i}^{j}+\log |d| .
$$

Given two variables $x, y$, consider the function

$$
[\cos x+\cos y+\cos (x-y)]^{n}, \quad n \geq 0,
$$

and its Fourier expansion

$$
[\cos x+\cos y+\cos (x-y)]^{n}=\sum_{r=0}^{\infty} \sum_{s=-\infty}^{\infty} c_{r, s}^{(n)} \cos (r x+s y)
$$

the set

$$
\mathcal{A}_{n}=\left\{(r, s) \in \mathbb{Z} \times \mathbb{Z} ; c_{r, s}^{(n)} \neq 0\right\}
$$

is finite. We have trivially $\mathcal{A}_{0}=\{(0,0)\}$ and $c_{0,0}^{(0)}=1$. It is easily seen that

$$
\mathcal{A}_{n} \subset \mathcal{A}_{n+1}, \text { for } n=1,2,3, \ldots \text {. }
$$


We can write

$$
[\cos x+\cos y+\cos (x-y)]^{n}=\sum_{(r, s) \in \mathcal{A}_{n}} c_{r, s}^{(n)} \cos (r x+s y),
$$

or more simply

$$
[\cos x+\cos y+\cos (x-y)]^{n}=\sum_{r, s} c_{r, s}^{(n)} \cos (r x+s y) .
$$

Estimate (8) then takes the form

$$
\log |R(a, b)|<\sum_{j=0}^{4} \alpha_{j} \sum_{r, s} c_{r, s}^{(j)} \sum_{i=1}^{c_{1}} \cos \frac{2 \pi(r a+s b)}{c}(2 i-1)+\log |d| .
$$

We also have

$$
\sum_{i=1}^{c_{1}} \cos \frac{2 \pi(r a+s b)}{c}(2 i-1)=\left\{\begin{array}{cl}
c_{1}(-1)^{2(r a+s b) / c} & \text { if } r a+s b \equiv 0 \quad\left(\bmod \frac{c}{2}\right) \\
0 & \text { if } r a+s b \neq 0 \quad\left(\bmod \frac{c}{2}\right) \\
& \text { and } c \equiv 0(\bmod 4) ; \\
-\frac{1}{2} \cos (r a+s b) \pi & \text { if } r a+s b \neq 0 \quad\left(\bmod \frac{c}{2}\right) \\
& \text { and } c \neq 0 \quad(\bmod 4) .
\end{array}\right.
$$

The next lemma guarantees that $r a+s b \not \equiv 0\left(\bmod \frac{c}{2}\right)$ for all $(r, s) \in \mathcal{A}_{4}$ with at most two exceptions. We denote by $(a, b)$ any solution of the congruence

$$
1+\zeta^{A}+\zeta^{B} \equiv 0 \quad(\bmod \mathbf{q}), A \not \equiv 0, B \not \equiv 0, A \not \equiv B \quad(\bmod c)
$$

the numbers $a, b$ are determined $\bmod c$. Relation (6) says that the set of the solutions to (11) is nonempty by hypothesis.

Lemma 2. Let $\mathcal{A}=\{(2,-4),(4,-2),(2,2)\}$. Then the following hold true:

(I) The pairs $(b, a),(-a, b-a)$ are also solutions of (11).

(II) The congruence

$$
r a+s b \equiv 0 \quad\left(\bmod \frac{c}{2}\right)
$$

is impossible for $(r, s) \in \mathcal{A}_{4}-\mathcal{A}-\{(0,0)\}$.

(III) If $c \not \equiv 0(\bmod 4)$, then congruence (12) is impossible for $(r, s) \in \mathcal{A}_{4}-\{(0,0)\}$, while if $c \equiv 0(\bmod 4)$, then congruence (12) can be satisfied by at most one $(r, s) \in$ $\mathcal{A}$ and in this case $2(r a+s b) / c$ is odd.

Proof. The first assertion of the lemma is obvious.

(II) We have $\mathcal{A}_{1}=\{(1,-1),(1,0),(0,1)\}$ and

$$
\begin{array}{cc}
\mathcal{A}_{2}= & \mathcal{A}_{1} \cup\{(0,0),(1,-2),(2,-2),(2,-1),(2,0),(1,1),(0,2)\}, \\
\mathcal{A}_{3}=\mathcal{A}_{2} \cup\{(1,-3),(2,-3),(3,-3),(3,-2),(3,-1),(3,0),(2,1),(1,2),(0,3)\}, \\
\mathcal{A}_{4}=\mathcal{A}_{3} \cup\{(1,-4),(2,-4),(3,-4),(4,-4),(4,-3), \\
& (4,-2),(4,-1),(4,0),(3,1),(2,2),(1,3),(0,4)\} .
\end{array}
$$

Obviously, the set $\mathcal{A}_{4}-\mathcal{A}-\{(0,0)\}$ consists of 27 elements.

Consider the transformations $\tau_{0}, \tau_{1}, \tau_{2}$ defined by

$$
\tau_{0}(a, b)=(a, b), \quad \tau_{1}(a, b)=(b, a), \quad \tau_{2}(a, b)=(-a, b-a) .
$$


All these transformations are of the form

$$
\tau_{i}(a, b)=\left(a_{11}^{(i)} a+a_{12}^{(i)} b, a_{21}^{(i)} a+a_{22}^{(i)} b\right), \quad i=0,1,2,
$$

or in matrix notation

$$
\tau_{i}(a, b)^{T}=\left(\begin{array}{ll}
a_{11}^{(i)} & a_{12}^{(i)} \\
a_{21}^{(i)} & a_{22}^{(i)}
\end{array}\right)\left(\begin{array}{c}
a \\
b
\end{array}\right), \quad a_{k l} \in \mathbb{Z} .
$$

The image $\tau_{i}(a, b)$ is also a solution of (11) for $i=0,1,2$ because of the part (I) of the lemma. For this reason, if

$$
r_{1} a+s_{1} a \not \equiv 0 \quad\left(\bmod \frac{c}{2}\right)
$$

for some $\left(r_{1}, s_{1}\right) \in \mathcal{A}_{4}-\mathcal{A}-\{(0,0)\}$ and for every solution $(a, b)$ of (11), then also

$$
r_{1}\left(a_{11}^{(i)} a+a_{12}^{(i)} b\right)+s_{1}\left(a_{21}^{(i)} a+a_{22}^{(i)} b\right) \not \equiv 0 \quad\left(\bmod \frac{c}{2}\right)
$$

for every $i=0,1,2,3$. Since the left member of 15 is equal to

$$
\left(r_{1} a_{11}^{(i)}+s_{1} a_{21}^{(i)}\right) a+\left(r_{1} a_{12}^{(i)}+s_{1} a_{22}^{(i)}\right) b
$$

it follows that if (14) is true for some $\left(r_{1}, s_{1}\right) \in \mathcal{A}_{4}-\mathcal{A}-\{(0,0\}$ and for every solution $(a, b)$, then the relation $r a+s b \not \equiv 0(\bmod c / 2)$ is also true for the pair $(r, s)$, where

$$
\left(\begin{array}{c}
r \\
s
\end{array}\right)=\left(\begin{array}{ll}
a_{11}^{(i)} & a_{21}^{(i)} \\
a_{12}^{(i)} & a_{22}^{(i)}
\end{array}\right)\left(\begin{array}{c}
r_{1} \\
s_{1}
\end{array}\right), \quad i=0,1,2 .
$$

A subset $\mathcal{B}$ of $\mathcal{A}_{4}-\mathcal{A}-\{(0,0)\}$ is called fundamental, if, for every pair $(r, s) \in$ $\mathcal{A}_{4}-\mathcal{A}-\{(0,0)\}$, the equality

$$
\left(\begin{array}{l}
r \\
s
\end{array}\right)= \pm T\left(\begin{array}{l}
r_{1} \\
s_{1}
\end{array}\right)
$$

holds true for some $\left(r_{1}, s_{1}\right) \in \mathcal{B}$ and for some transformation $T$ composed of the transformations (16).

The final conclusion of the above discussion is the following: To prove part (II) of Lemma 2, it suffices to prove that the congruence (12) is impossible for all $(r, s) \in \mathcal{B}$, where $\mathcal{B}$ is a fundamental subset of $\mathcal{A}_{4}-\mathcal{A}-\{(0,0)\}$. A simple calculation shows that a fundamental subset of $\mathcal{A}_{4}-\mathcal{A}-\{(0,0)\}$ is the following

$$
\mathcal{B}=\{(1,0),(2,0),(3,0),(4,0),(1,1),(1,-3),(1,-4)\}
$$

We distinguish two cases (A), (B).

(A) $(r, s) \in\{(1,0),(2,0),(3,0),(4,0)\}$; we have to prove that

$$
a \not \equiv 0,2 a \not \equiv 0,2^{2} a \not \equiv 0,3 a \not \equiv 0 \quad\left(\bmod \frac{c}{2}\right) .
$$

We prove the first three relations by induction on the exponents of the powers $1,2,2^{2}$. The first relation is true by hypothesis. Assuming that $2^{j} a \not \equiv 0(\bmod c / 2)$, let us prove that $2^{j+1} a \not \equiv 0(\bmod c / 2)$. Indeed, the contrary hypothesis $2^{j+1} a \equiv 0$ $(\bmod c / 2)$ implies that $2^{j+1} a=k(c / 2)$, where $k$ is an integer. The number $k$ is odd, because if $k$ were even, then this fact would vitiate the induction hypothesis; 
in consequence, $c$ is divisible by 4 and so $a=k\left(c / 2^{j+2}\right)$. Then $\zeta^{a}=\xi$, where $\xi$ is a primitive $2^{j+2}$-th root of unity, and congruence (6) becomes

$$
1+\xi \equiv-\zeta^{b} \quad(\bmod \mathbf{q}) \text {. }
$$

Congruence (17) implies then $(1+\xi)^{c} \equiv 1(\bmod \mathbf{q})$ and taking norms we conclude that $2^{c} \equiv 1(\bmod q)$, which is impossible by hypothesis.

It remains to prove that $3 a \not \equiv 0(\bmod c / 2)$; indeed, if were $3 a \equiv 0(\bmod c / 2)$ this would imply $($ since $(3, c)=1)$ that $a \equiv 0(\bmod c / 2)$, which is impossible by hypothesis.

(B) $(r, s) \in\{(1,1),(1,-3),(1,-4)\}$; assume that the congruence (12) holds true for such a pair $(r, s)$. We shall prove that this leads to a contradiction. We have by hypothesis

$$
\zeta^{r a} \equiv \pm \zeta^{-s b} \quad(\bmod \mathbf{q}), \quad 1+\zeta^{a}+\zeta^{b} \equiv 0 \quad(\bmod \mathbf{q}) .
$$

It follows that at least one of the polynomials

$$
f_{r, s}^{ \pm}(t)= \begin{cases}(1+t)^{r} \pm t^{-s} & \text { if } s<0, \\ t^{s}(1+t)^{r} \pm 1 & \text { if } s>0,\end{cases}
$$

has a common root $\bmod q$ with the polynomial $t^{c}-1=\left(t^{c / 2}-1\right)\left(t^{c / 2}+1\right)$. This implies that at least one of the congruences

$$
R\left(f_{r, s}^{ \pm}(t), t^{c / 2}+(-1)^{n}\right) \equiv 0 \quad(\bmod q)
$$

holds true for every $n \in\{1,2\}$. If $d_{r, s}^{ \pm}$are the degrees of the polynomials (19) and $\rho_{1}^{ \pm}, \rho_{2}^{ \pm}, \ldots$, their roots, then

$$
R\left(f_{r, s}^{ \pm}(t), t^{c / 2}+(-1)^{n}\right)=\prod_{i=1}^{d_{r, s}^{ \pm}}\left[\rho_{i}^{c / 2}+(-1)^{n}\right]
$$

We have to distinguish between two cases (a) and (b):

(a) $(r, s)=(1,1)$; we have

$$
\begin{gathered}
f_{1,1}^{ \pm}(t)=t^{2}+t \pm 1 \\
0<\left|R\left(t^{2}+t+1, t^{c / 2}+(-1)^{n}\right)\right| \leq 4 \\
R\left(t^{2}+t-1, t^{c / 2}+(-1)^{n}\right)=\left[\left(-\omega_{1}\right)^{\frac{c}{2}}+(-1)^{n}\right] \cdot\left[\left(-\omega_{2}\right)^{\frac{c}{2}}+(-1)^{n}\right] \\
=1+(-1)^{c / 2}+(-1)^{n+\frac{c}{2}} L_{c / 2} \neq 0 .
\end{gathered}
$$

Relation (21) contradicts hypothesis (4). Each of the numbers (22) divides by part (ii) of Lemma 1 the number $u_{c}$ for $n=1,2$. Congruence (20) leads then, in view of part (iii) of Lemma 1, to a contradiction.

(b) $(r, s) \in\{(1,-3),(1,-4)\}$; we have

$$
f_{1,-3}(t)= \pm t^{3}+t+1 \text { and } f_{1,-4}(t)= \pm t^{4}+t+1 \text {. }
$$

For $c \geq 22$, a simple calculation shows that

$$
0<\left|R\left(f_{r, s}^{ \pm}(t), t^{c / 2}+(-1)^{n}\right)\right|<\theta^{c / 4}
$$

for $(r, s) \in\{(1,-3),(1,-4)\}$, which contradicts, in view of (20), hypothesis (4). 
(III) If two of the congruences

$$
2 a-4 b \equiv 0,4 a-2 b \equiv 0,2 a+2 b \equiv 0 \quad\left(\bmod \frac{c}{2}\right),
$$

were true, then for these two congruences, say for the first and for the second, we would have

$$
\begin{aligned}
0 \equiv & (2 a-4 b)+(4 a-2 b) \equiv 6 a-6 b \quad\left(\bmod \frac{c}{2}\right) \Rightarrow 6 a-6 b=k \frac{c}{2} \\
& \Rightarrow 2 a-2 b=k_{1} \frac{c}{2} \quad(\text { because } c \neq 0 \quad(\bmod 3)) \\
& \Rightarrow 2 a-2 b \equiv 0 \quad\left(\bmod \frac{c}{2}\right),
\end{aligned}
$$

which is absurd, since $(2,-2) \in \mathcal{A}_{4}-\mathcal{A}-\{(0,0)\}$. If one of the congruences [23] is true, this means that

$$
2 a-4 b \equiv 0 \quad \text { or } 4 a-2 b \equiv 0 \quad \text { or } 2 a+2 b \equiv 0 \quad\left(\bmod \frac{c}{2}\right)
$$

or equivalently

$$
2 a-4 b=k_{1} \frac{c}{2} \quad \text { or } \quad 4 a-2 b=k_{2} \frac{c}{2} \quad \text { or } \quad 2 a+2 b=k_{3} \frac{c}{2} .
$$

The integers $k_{1}, k_{2}, k_{3}$ cannot be even; otherwise this would imply that

$$
a-2 b \equiv 0 \text { or } 2 a-b \equiv 0 \text { or } a+b \equiv 0 \quad\left(\bmod \frac{c}{2}\right) \text {, }
$$

which is absurd, because $(1,-2),(2,-1),(1,1) \in \mathcal{A}_{4}-\mathcal{A}-\{(0,0)\}$. In case $c \not \equiv 0$ $(\bmod 4)$ the equalities $(24)$ are all impossible because the right members are odd numbers.

We then turn to the proof of theorem. We distinguish two cases $(\mathrm{A})$ and $(\mathrm{B})$. (A) $c \equiv 0(\bmod 4) ;$ then $c_{1}=\frac{c}{4}$ and $d=1$. In case the congruence $r a+s b \equiv 0$ $\left(\bmod \frac{c}{2}\right)$ holds true for one (and only one) $(r, s) \in \mathcal{A}$, it follows by Lemma 2 and relations (9), (10) that

$$
\log |R(a, b)|<\left[\alpha_{0} c_{0,0}^{(0)}+\alpha_{1} c_{0,0}^{(1)}+\alpha_{2} c_{0,0}^{(2)}+\alpha_{3} c_{0,0}^{(3)}+\alpha_{4}\left(c_{0,0}^{(4)}-c_{r, s}^{(4)}\right)\right] \frac{c}{4} .
$$

Since

$$
c_{0,0}^{(0)}=1, c_{0,0}^{(1)}=0, c_{0,0}^{(2)}=\frac{3}{2}, c_{0,0}^{(3)}=\frac{3}{2}, c_{0,0}^{(4)}=\frac{45}{8},
$$

and

$$
c_{r, s}^{(4)}=\frac{3}{4} \text { for }(r, s) \in \mathcal{A}
$$

we obtain the estimate

$$
\log |R(a, b)|<(0.809283336 \ldots) \frac{c}{4}<\frac{c}{4} \log \theta .
$$

In case the congruence $r a+s b \equiv 0\left(\bmod \frac{c}{2}\right)$ is impossible for all $(r, s) \in \mathcal{A}$, Lemma 2 , together with the relations (9), (10), imply the estimate

$$
\log |R(a, b)|<\left[\sum_{j=0}^{4} \alpha_{j} c_{0,0}^{(j)}\right] \frac{c}{4}=\frac{c}{4} \log \theta .
$$

Both estimates (25) and (26) contradict, by (7), hypothesis (4). 
(B) $c \not \equiv 0(\bmod 4) ;$ then $c_{1}=\frac{c}{4}-\frac{1}{2}, d=1+(-1)^{a}+(-1)^{b}$, and it follows by Lemma 2 and relations (9), (10) that

$$
\begin{aligned}
\log |R(a, b)| & <\sum_{j=0}^{4} \alpha_{j}\left[\frac{c_{1}}{4} c_{0,0}^{(j)}-\frac{1}{2} \sum_{\substack{r, s \\
(r, s) \neq(0,0)}} c_{r, s}^{(j)} \cos (r a+s b) \pi\right]+\log |d| \\
& =\sum_{j=0}^{4} \alpha_{j}\left[\frac{c}{4} c_{0,0}^{(j)}-\frac{1}{2} \sum_{r, s} c_{r, s}^{(j)} \cos (r a+s b) \pi\right]+\log |d| \\
& =\left[\sum_{j=0}^{4} \alpha_{j} c_{0,0}^{(j)}\right] \frac{c}{4}-\frac{1}{2} \sum_{j=0}^{4} \alpha_{j}\left[(-1)^{a}+(-1)^{b}+(-1)^{a-b}\right]^{j}+\log |d| .
\end{aligned}
$$

Hence

$$
\log |R(a, b)|<\left\{\begin{array}{l}
\frac{c}{4} \log \theta+\log |d|-0.01889 \text { if } a, b \text { are both even, } \\
\frac{c}{4} \log \theta-0.4103 \text { otherwise }
\end{array}\right.
$$

which by (7) contradicts hypothesis (4), since $q$ cannot divide the integer $d$.

\section{The Large Prime Divisors of $W_{c}$}

Let $c \geq 2$ be an integer such that $(3, c)=1$. A prime divisor $q$ of $W_{c}$ is called large if $q>\theta^{c / 4}$. Denote by $\mathcal{P}_{c}$ the set of large prime divisors of $W_{c}$; denote also by $P_{c}, Q_{c}, U_{c}$ (or, for simplicity, by $P, Q, U$ ) the largest prime divisor of the numbers $2^{c / 2}-1,2^{c / 2}+1, L_{c / 2}$, respectively. The set $\mathcal{P}_{c}$ is empty in case $c \equiv 4(\bmod 8)$, except when $c=20$. We can easily determine the set $\mathcal{P}_{c}$ using Theorem 3 in combination with the tables in [2] and [3]. Thus, in Table 2 below we list the large prime divisors of $W_{c}$ for all $c \leq 662$, such that $c \not \equiv 0(\bmod 3)$ and $c \not \equiv 4(\bmod 8)$ (the case $c=20$ is also included). We did not try to extend Table 2 beyond the value $c=662$, because for $c>662$, in the tables in [2] and [3] appear incomplete factorizations of the numbers (31), involving composite factors whose prime factors are unknown. We found that all the numbers in Table 2 are congruent to $1(\bmod c)$. We also found that for $c \leq 662$, and $q \in \mathcal{P}_{c}$, the number $(q-1) / c$ is always composite except when

$$
(c, q) \in\{(10,31),(20,61),(22,683)\} .
$$

The verification of the last assertion has been carried out without much difficulty because in almost all cases, the numbers $(q-1) / c$ were found to have a small prime divisor. The only difficulties arose from the numbers $P_{482}, Q_{362}, Q_{454}$. Indeed we found that the least prime divisor of the numbers $\left(P_{482}-1\right) / 482$ and $\left(Q_{362}-1\right) / 362$ is 21221 and 412987, respectively, while the converse of Fermat's Theorem with base 2 showed that the number

$$
\left(Q_{454}-1\right) / 454=154145750348602301130214857398044121373127
$$

is composite (with unknown factors). 
TABLE 2. The large prime divisors of $W_{c}$ for $c \leq 662$

\begin{tabular}{|c|c|c|c|c|c|c|c|}
\hline$c$ & $\mathcal{P}_{c}$ & $\bar{c}$ & $\mathcal{P}_{c}$ & $c$ & $\mathcal{P}_{c}$ & $c$ & $\mathcal{P}_{c}$ \\
\hline 2 & $Q U$ & 166 & $P$ & 334 & $\begin{array}{ll}P & Q\end{array}$ & 502 & $Q$ \\
\hline 8 & $\bar{Q}$ & 170 & $\begin{array}{ll}P & Q\end{array}$ & 338 & $P$ & 506 & $\varnothing$ \\
\hline 10 & $\begin{array}{lll} & P & Q\end{array}$ & 176 & $\varnothing$ & 344 & $\varnothing$ & 512 & $\bar{Q}$ \\
\hline 14 & $\begin{array}{lll}P & Q & U\end{array}$ & 178 & 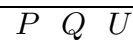 & 346 & $\varnothing$ & 514 & $\varnothing$ \\
\hline 16 & $Q$ & 182 & $\varnothing$ & 350 & $\varnothing$ & 518 & $P$ \\
\hline 20 & 61 & 184 & $Q$ & 352 & $\bar{Q}$ & 520 & $\varnothing$ \\
\hline 22 & $\begin{array}{lll}P & Q & U\end{array}$ & 190 & $\bar{Q}$ & 358 & $\begin{array}{ll}P & Q\end{array}$ & 526 & $\bar{Q}$ \\
\hline 26 & $\begin{array}{lll}P & Q & U\end{array}$ & 194 & $\begin{array}{ll}P & Q\end{array}$ & 362 & $\begin{array}{ll}P & Q\end{array}$ & 530 & $\varnothing$ \\
\hline 32 & $Q$ & 200 & $\varnothing$ & 368 & $\varnothing$ & 536 & $Q$ \\
\hline 34 & $\begin{array}{lll} & Q & U\end{array}$ & 202 & $\bar{Q}$ & 370 & $\bar{Q}$ & 538 & $\begin{array}{ll}P Q \\
\end{array}$ \\
\hline 38 & $\begin{array}{lll} & Q & U\end{array}$ & 206 & $\begin{array}{ll}P Q \\
\end{array}$ & 374 & $\begin{array}{ll}P & Q\end{array}$ & 542 & $P$ \\
\hline 40 & $\bar{Q}$ & 208 & $\bar{Q}$ & 376 & $\varnothing$ & 544 & $\varnothing$ \\
\hline 46 & $P Q$ & 214 & $\begin{array}{ll}P & Q\end{array}$ & 382 & $Q$ & 550 & $Q$ \\
\hline 50 & $\varnothing$ & 218 & $\begin{array}{ll}P & Q\end{array}$ & 386 & $\varnothing$ & 554 & $Q$ \\
\hline 56 & $\bar{Q}$ & 224 & $\varnothing$ & 392 & $\varnothing$ & 560 & $\varnothing$ \\
\hline 58 & $\bar{Q}$ & 226 & $\bar{U}$ & 394 & $P$ & 562 & $\begin{array}{ll}P Q \\
\end{array}$ \\
\hline 62 & $\begin{array}{lll}P & Q & U\end{array}$ & 230 & $\varnothing$ & 398 & $\begin{array}{ll}P & Q\end{array}$ & 566 & $\begin{array}{l}P Q \\
P Q\end{array}$ \\
\hline 64 & $Q$ & 232 & $Q$ & 400 & $\varnothing$ & 568 & $Q$ \\
\hline 70 & $\varnothing$ & 238 & $\varnothing$ & 406 & $P$ & 574 & $P$ \\
\hline 74 & $\begin{array}{lll}P & Q & U\end{array}$ & 242 & $\begin{array}{lll}P & Q & U\end{array}$ & 410 & $\bar{Q}$ & 578 & $P$ \\
\hline 80 & $Q$ & 248 & $\varnothing$ & 416 & $\varnothing$ & 584 & $Q$ \\
\hline 82 & $\begin{array}{lll} & Q & U\end{array}$ & 250 & $\varnothing$ & 418 & $\varnothing$ & 586 & $P$ \\
\hline 86 & $\bar{Q}$ & 254 & $\begin{array}{ll}P & Q\end{array}$ & 422 & $P$ & 590 & $Q$ \\
\hline 88 & $\bar{Q}$ & 256 & $\varnothing$ & 424 & $\varnothing$ & 592 & $Q$ \\
\hline 94 & $Q U$ & 262 & $P Q$ & 430 & $Q$ & 598 & $Q$ \\
\hline 98 & $\begin{array}{lll}P & Q & U\end{array}$ & 266 & $\begin{array}{ll}P & Q\end{array}$ & 434 & $\bar{Q}$ & 602 & $\bar{Q}$ \\
\hline 104 & $\varnothing$ & 272 & $\varnothing$ & 440 & $\varnothing$ & 608 & $\varnothing$ \\
\hline 106 & $Q U$ & 274 & $\varnothing$ & 442 & $\begin{array}{ll}P & Q\end{array}$ & 610 & $\varnothing$ \\
\hline 110 & $\varnothing$ & 278 & $\begin{array}{ll}P Q \\
\end{array}$ & 446 & $\begin{array}{l}Q U \\
Q\end{array}$ & 614 & $P$ \\
\hline 112 & $\bar{Q}$ & 280 & $\bar{Q}$ & 448 & $\varnothing$ & 616 & $\bar{Q}$ \\
\hline 118 & $P$ & 286 & $\varnothing$ & 454 & $P Q$ & 622 & $P \quad Q \quad U$ \\
\hline 122 & $\begin{array}{lll}P & Q & U\end{array}$ & 290 & $P Q$ & 458 & $Q \quad U$ & 626 & $Q \quad U$ \\
\hline 128 & $\bar{Q}$ & 296 & $\bar{Q}$ & 464 & $\bar{Q}$ & 632 & $\bar{Q}$ \\
\hline 130 & $P$ & 298 & $Q$ & 466 & $P Q$ & 634 & $Q$ \\
\hline 134 & $P Q$ & 302 & $\bar{Q}$ & 470 & $\varnothing$ & 638 & $P$ \\
\hline 136 & $Q$ & 304 & $\varnothing$ & 472 & $Q$ & 640 & $\varnothing$ \\
\hline 142 & $Q U$ & 310 & $\varnothing$ & 478 & $P Q$ & 646 & $P$ \\
\hline 146 & $P Q$ & 314 & $\varnothing$ & 482 & $P Q$ & 650 & $P$ \\
\hline 152 & $Q$ & 320 & $Q$ & 488 & $\varnothing$ & 656 & $\varnothing$ \\
\hline 154 & $P$ & 322 & $\bar{Q}$ & 490 & $P$ & 658 & $P Q$ \\
\hline 158 & $Q U$ & 326 & $\varnothing$ & 494 & $Q$ & 662 & $P$ \\
\hline 160 & $\varnothing$ & 328 & $\bar{Q}$ & 496 & $\bar{Q}$ & & \\
\hline
\end{tabular}

\section{Applications to Fermat's congruence}

Let $p, q$ be odd primes. It is easy to prove that Fermat's congruence (10) has a nontrivial solution if $q \not \equiv 1(\bmod p)$ or $(3, c)>1$. However, the case $q \equiv 1(\bmod p)$, 
$(3, c)=1$ involves many difficult and still unsolved problems. Combining together Theorems 1 and 3 we obtain the following main result.

Theorem 4. Let $p, q$ be odd primes such that $(p, q) \neq(3,61)$. Then Fermat's congruence

$$
x^{p}+y^{p}+z^{p} \equiv 0 \quad(\bmod q)
$$

has only trivial solutions (that is, solutions $(x, y, z)$ such that $x y z \equiv 0(\bmod q))$ provided that:

(i) $q=1+c p$ and $(3, c)=1$;

(ii) $2^{c} \not \equiv 1(\bmod q)$, or $c \equiv 0(\bmod 4)$;

(iii) $L_{c / 2} \not \equiv 0(\bmod q)$, or $c \equiv 0(\bmod 4)$;

(iv) $q>\theta^{c / 4}$.

The stronger condition $c \equiv 0(\bmod 4)$ in (ii) instead of $c \equiv 0(\bmod 8)$, is due to the fact that the number $2^{c / 2}+1$ does not have prime divisors of the form $q \equiv 1(\bmod 8)$; this has been proved in [14, p. 170]. Theorem 4 improves upon the previous results of Vandiver [15], Krasner [10] and the author [14].

The numerical evidence indicates that the conditions

$$
2^{c} \not \equiv 1 \quad(\bmod q) \quad \text { and } \quad L_{c / 2} \not \equiv 0 \quad(\bmod q)
$$

are almost always superfluous; more precisely:

Proposition 1. Let $p, q$ be odd primes. Then, congruence (27) has only trivial solutions for every prime exponent

$$
p \leq \frac{\theta^{166}-1}{664}=(3.9769287 \ldots) 10^{55},
$$

provided that $q=1+c p,(3, c)=1, q>\theta^{c / 4}$ and that

$$
(p, q) \neq(3,31),(3,61),(31,683) .
$$

Proof. Assume that the pair $(p, q)$ contradicts the truth of the proposition. Then, necessarily, $q \in \mathcal{P}_{c}$. By the results in Section 4 (last paragraph) it follows that $c \geq 664$. In consequence

$$
p>\frac{\theta^{c / 4}-1}{c} \geq \frac{\theta^{166}-1}{664},
$$

which is impossible by hypothesis.

Proposition 1 leads naturally to the following conjecture.

Conjecture 1. Let $p, q$ be odd primes. Then, congruence (27) has only trivial solutions provided that $q=1+c p,(3, c)=1, q>\theta^{c / 4}$ and that $(p, q) \neq$ $(3,31),(3,61),(31,683)$.

It is important to note that inequality $q>\theta^{c / 4}$ is equivalent to

$$
\begin{aligned}
q & <\frac{4}{\log \theta} p \log p+\frac{4}{\log \theta} p \log g \log p \\
& =(4.936 \ldots) p \log p+(4.936 \ldots) p \log \log p
\end{aligned}
$$

(in fact, the last inequality is a bit weaker). According to a classical result of Dickson, congruence (27) has nontrivial solutions if

$$
q>(p-1)^{2}(p-2)^{2}+6 p-2 .
$$


Chowla 4] conjectured that the stronger inequality $q>p^{2}$ holds true for sufficiently large $p$.

\section{ACKNOWLEDGMENT}

I would like to express my thanks to the referee for valuable suggestions.

\section{REFERENCES}

[1] D.W. Boyd, The asymptotic behaviour of the circulant determinant, J. Math. Appl. 86 (1982), 30-38. MR 83f:10007

[2] J. Brillhart, D.H. Lehmer, J.L. Selfridge, B. Tuckerman and S.S. Wagstaff Jr., Factorizations of $b^{n} \pm 1, b=2,3,5,6,7,10,11,12$ up to high powers, Contemporary Mathematics 22, American Mathematical Society, Providence, 1988. MR 90d:11009

[3] J. Brillhart, P.L. Montgomery and R.D. Silverman, Tables of Fibonacci and Lucas Factorizations, Math. Comp. 50 (1988), 251-260. MR 89h:11002

[4] S. Chowla, Some conjectures in elementary number theory, Norske Vid. Selsk. Forh. (Trondheim) 35 (1962), 13. MR 25:2995

[5] P. Dénes, An extension of Legendre's criterion in connection with the first case of Fermat's Last Theorem, Publ. Math. Debrecen 2 (1951), 115-120. MR 13:822h

[6] G. Fee and A. Granville, The prime factors of Wendt's binomial circulant determinant, Math. Comp. 57 (1991), 839-848. MR 92f:11183

[7] D. Ford and V. Jha, On Wendt's Determinant and Sophie Germain's Theorem, Experimental Math. 2 (1993), 113-119. MR 95b:11029

[8] J.S. Frame, Factors of the binomial circulant determinant, Fibonacci Quart. 18 (1980), 9-23. MR 81j:11007.

[9] C. Helou, On Wendt's determinant, Math. Comp. 66 (1997), 1341-1346. MR 97j:11014

[10] M. Krasner, A propos du critère de Sophie Germain - Furtwängler pour le premier cas du théorèm de Fermat, Mathematica Cluj. 16 (1940), 109-114. MR 1:291k

[11] E. Lehmer, On a resultant connected with Fermat's Last Theorem, Bull. Amer. Math. Soc. 41 (1935), 864-867.

[12] P. Ribenboim, 13 Lectures on Fermat's Last Theorem, Springer, New York, 1979. MR 81f: 10023

[13] A. Simalarides, Applications of the theory of cyclotomic field to Fermat's equation and congruence, Ph.D. Thesis, Athens University, Athens 1984.

[14] A. Simalarides, Sophie Germain's Principle and Lucas numbers, Math. Scand. 67 (1990), 167-176. MR 92c:11026

[15] H.S. Vandiver, Some theorems in finite field theory with applications to Fermat's Last Theorem, Proc. Nat. Acad. Sci. U.S.A. 30 (1944), 362-367. MR 6:117e

[16] E. Wendt, Arithmetische Studien über den letzten Fermatschen Satz, welcher aussagt, dass die Gleichung $a^{n}=b^{n}+c^{n}$, für $n>2$ in ganzen Zahlen nicht auflösbar ist, J. Reine Angew. Math. 113 (1894), 335-347.

T.E.I. of Chalcis, Psahna 34400, Euboea, Greece 